\title{
The influence of structural defects on the optical properties of synthetic opals
}

\author{
Yevchik A., Moiseyenko V. and Dergachov M. \\ Oles Honchar Dnipropetrovsk National University, Dnipropetrovsk, Ukraine. \\ e-mail: av.yevchik@gmail.com
}

Received: 10.12.2014

\begin{abstract}
We demonstrate the influence of structural defects present in opals on the Bragg reflection, the fluorescence and the Raman spectra. Based on the real opal structure, the relative ordering parameter is suggested as a criterion of sample quality. A 1D model of a periodic layered structure containing the defects typical for the synthetic opals is developed. Using a comparison of experimental and simulated reflection spectra, we show that the model can be brought closer to the real conditions if the elastic multiple scattering is taken into account within the model. To study the influence of defects on the spontaneous emission and the inelastic scattering, we have measured the fluorescence spectra of the dye R6G and the Raman spectra of $\mathrm{Bi}_{12} \mathrm{SiO}_{20}$ incorporated in the synthetic opal. Amplification of both the fluorescence intensity for R6G and the Raman intensity for $\mathrm{Bi}_{12} \mathrm{SiO}_{20}$ is observed for synthetic opal templates.
\end{abstract}

Keywords: synthetic opals, structural defects, relative ordering parameter, R6G, amplification of optical spectra, light localization, multiple elastic scattering.

PACS: $78.67 . \pm \mathrm{n}, 78.20 . \mathrm{Bh}$

UDC: $535.3+538.9$

\section{Introduction}

Synthetic opals (SOs) [1-3] represent a class of 3D photonic crystals (PCs) obtained by a selfassembly method. These materials offer great opportunities for studying the effects of spontaneous emission enhancement, low-threshold lasing, information processing and transferring [4], light channelling, and light localization. Considering the effects mentioned above, they are also interesting for creating various integral optical devices.

Fabrication of these SO-based devices requires incorporation of structural defects in a controlled way. The structure of the initial SOs composed of $\mathrm{SiO}_{2}$ globules and interglobular air spaces is not completely regular and includes various types of uncontrolled defects [5, 6]. The presence of single defects in such structures generates local photonic states and, as a consequence, nonzero integral density of optical states (DOS) in the photonic band gap, similarly to the case of semiconductors. The number of the local photonic states increases with increasing amount of defects, up to complete disappearance of the bandgap [7]. From the applied point of view, regular structures with single defects can be used for channelling light in optical waveguides, splitters and multiplexers [8-10]. In the structures characterized with a certain disorder degree, one can expect the effect of photon localization predicted earlier $[11,12]$. When the concentration of uncontrolled defects becomes high enough, the SOs are interesting for studies of random-lasing effects [13]. Thus, there is a clear need in establishing objective criteria to identify applicability of the SOs in one or another research branch.

The aim of this work is to study theoretically and experimentally the physical reasons for the influence of structural defects on the optical properties of globular PCs based on the SOs. 


\section{Classification of structural defects of SO samples}

Bulk SO samples were grown using natural sedimentation of $\mathrm{SiO}_{2}$ globules suspension. Synthesis of silica globules was performed with a modified Stöber method [14]. Dimensions of the samples thus obtained were about $1.0 \times 1.0 \times 0.5 \mathrm{~cm}^{3}$.

The initial opals were characterized by analyzing the surface structure with an X-Ray Microanalyzer JEO JXA 8200 and by measuring the optical transmission and reflection spectra in the visible spectral range.

Any real SO samples always have some portion of structural defects. The microscopic analysis has shown that the SO samples contain such structural defects as vacancies (see label 1 in Fig. 1a), or a local disorder consisting in violation of a local-range order at the distances ranging from a few to several hundred lattice parameters (label 2 in Fig. 1a). 3D defects are also present, i.e. micro-pits and cracks (see labels 3 and 4 in Fig. 1a). Moreover, there are spatial regions where the globules have different sizes (labels 5 in Fig. 1a). These defects represent a class of microscopic defects, with their sizes being compared to the lattice parameter of the SO. On the other hand, the defects such as cracks, pits or differently oriented domains represent a class of macroscopic defects if only their sizes are significantly larger than the wavelength of light (see Fig. 1b).

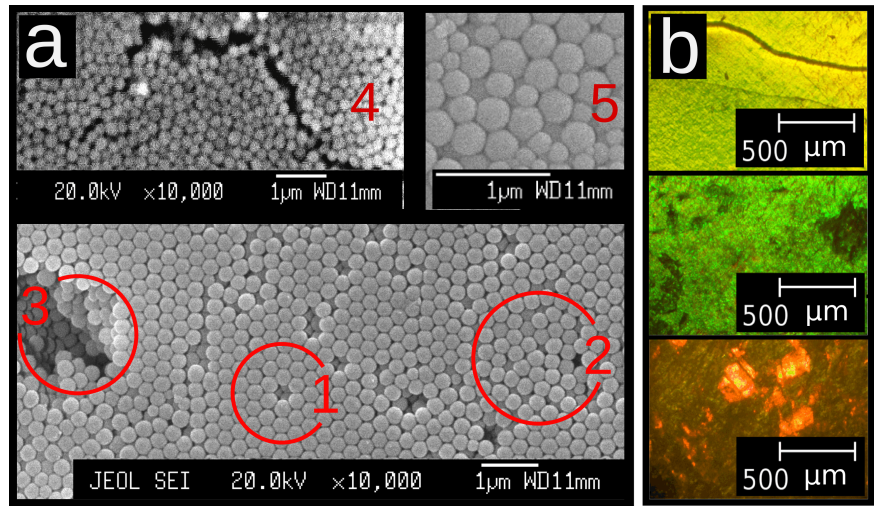

Fig. 1. Photomicrographs of SO surface with defective inclusions: vacancies (1), local disorders (2), pits (3), cracks (4), and different globule sizes (5), as obtained with the X-Ray microanalyzer JEO JXA 8200 (a); Photos of surface of the SO including macroscopic defects, as obtained with the optical microscope (b).

Disorientation of the (111) planes of differently oriented domains relative to the average orientation of the growth plane can be as large as $\leq \pm 5^{\circ}$. Additionally, regular alternation of the layers with hexagonal packing is randomly disturbed along the direction of growth axis $<111>$. As a result, the areas with different brightness degree of irisation are present in the SO samples. This results in decreasing coefficient of Bragg reflection from the system of (111) planes, as well as in increasing part of the diffused reflection.

The Bragg law we use for optical characterization of our samples is expressed as follows:

$$
\lambda_{0}(\theta)=2 d \sqrt{\varepsilon_{e f f}-\sin ^{2} \theta} .
$$

Here the globule diameter $D$ and the distance $d$ between the (111) planes (Note that the fcc structure of the SO samples leads to the relationship $D=d \sqrt{3 / 2}$ ) are determined by the peak position $\lambda_{0}$ in the Bragg reflection spectra or the minimum position in the transmission spectra, and $\varepsilon_{\text {eff }}$ stands for the effective dielectric permittivity. The latter is determined by the sum of 
contributions of the dielectric permittivities $\varepsilon_{i}$ of substances incorporated in the SO pores, according to their volume fractions $f_{i}: \varepsilon_{\text {eff }}=0.74 \times \varepsilon_{\mathrm{SiO}_{2}}+\sum_{i} f_{i} \times \varepsilon_{i}$, with $\sum_{i} f=0.26$.

The initial SO samples have a band gap, a so-called stop-band, only along certain spatial directions. The spectral width and the position of the photonic stop-band are given by the position $\lambda_{0}$ and the halfwidth $\Delta \lambda$ of the Bragg reflection band. The position of the stop-band corresponds to the region 535-620 $\mathrm{nm}$ along the $<111>$ direction for the initial SO samples.

Investigation of structural defects in the SOs needs a parameter quantifying structural ordering degree for a sample to be introduced. Suppose that the irisation areas in the SO sample volume under test are distributed uniformly, and their number coincides with the number of areas preserving a regular structure. Then the relative amount of the ordered areas on the surface should reflect the relative amount of the ordered areas inside the volume. We express the relative ordering parameter as a ratio of the total surface of the ordered areas, $S_{\text {order }}$, to the overall area of the sample surface, $S_{\text {overall }}$ :

$$
\eta_{\text {order }}=S_{\text {order }} / S_{\text {overall }} .
$$

The average areas $S_{\text {order }}$ and $S_{\text {overall }}$ can be estimated using computer processing of surface microphotographs and a graphical editor.

\section{The model of $1 \mathrm{D}$ layered structure with defects}

In order to compare experimental and calculation results for the influence of defects on the optical spectra of opals, we have developed a model of a 1D periodic structure involving the defects typical for the SO. The reflection spectra $R(\lambda)$ have been simulated basing on a transfer-matrix technique [15]. Simulations of the $\operatorname{DOS}(\lambda)$ spectra have been performed using a plane-wave expansion technique [16-18].

\subsection{Description of our model: the approach and basic parameters}

The period $d$ of the structure and the size $D$ of the structural element associated with the globular diameter can be expressed as $d=d_{0} \pm \Delta \times d_{\text {disamp }}$ and $D=D_{0} \pm \Delta \times D_{\text {disamp }}$. Here $d_{0}$ denotes the period of regular structure, $d_{\text {disamp }}$ (see Fig. 2) the maximum amplitude of deviation from $d_{0}, D_{0}$ the value associated with the globular diameter corresponding to the maximum of distribution function of globule diameters, $D_{\text {disamp }}$ the maximum deviation from this diameter, and $\Delta$ can take random values in the range from -1 to +1 .

Simulations have been carried out using a mathematical package GNU Octave. The simulation datasets $\operatorname{DOS}(\lambda)$ and the $R(\lambda)$ have been created while iterating sequentially the light wavelength $\lambda$ in the range of 350-650 nm. For higher accuracy, 1000 iteration cycles have been run, with subsequent averaging of the spectra. Combining the cycles with $d=d_{0}\left(D=D_{0}\right)$ and those with $d=d_{0} \pm \Delta \times d_{\text {disamp }}$ ( $D=D_{0} \pm \Delta \times D_{\text {disamp }}$ ) has allowed us to introduce the relative ordering parameter $\eta_{\text {order }}$. It is determined by the ratio of the number of cycles that result in the regular structure, $C_{\text {order }}$, to the total cycle number, $C_{\text {overall }}: \eta_{\text {order }}=C_{\text {order }} / C_{\text {overall }}$. When comparing the calculation results with the experimental data for real samples, further adaptation of our model is needed. This can be achieved after assigning the values taken from the real samples to the $d_{\text {disamp }}, D_{\text {disamp }}$ and $\eta_{\text {order }}$ parameters. 

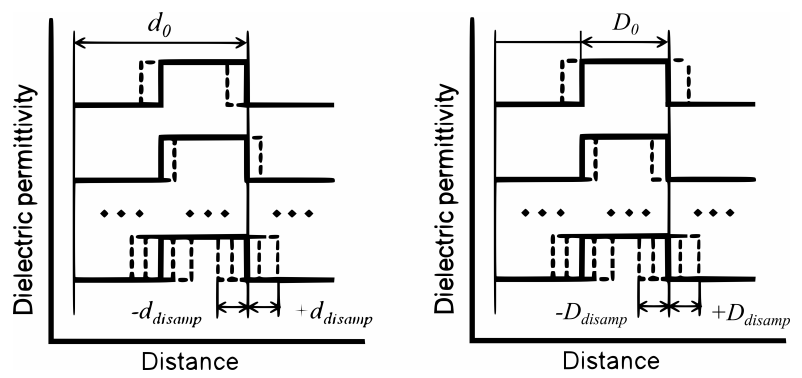

Fig. 2. A scheme illustrating definitions of the model parameters $d_{\text {disamp }}$ and $D_{\text {disamp }}$.

\subsection{Reflection spectra: simulations and experiments}

We have simulated the reflection spectra for the multilayer structure formed by sequentially arranged pairs of layers characterized with the parameters $d$ and $D$, and the refractive indices $n_{1}$ and $n_{2}$. The normal incidence of light has been considered. Here the effect of the defects can be taken into account by assigning the quantities obtained from the model to the $d$ and $D$ parameters.

The reflection spectra simulated and measured by us yield in the dependence of the relative reflection-band halfwidth and the value $\eta_{\text {order }}$ (see Fig. 3 a). Here curves 1 and 3 correspond respectively to simulation and experiment. The clear differences of these dependences are easily understood since the model is incapable to account for the impact of elastic multiple scattering occurring at the globules with diameters close to the light wavelength.
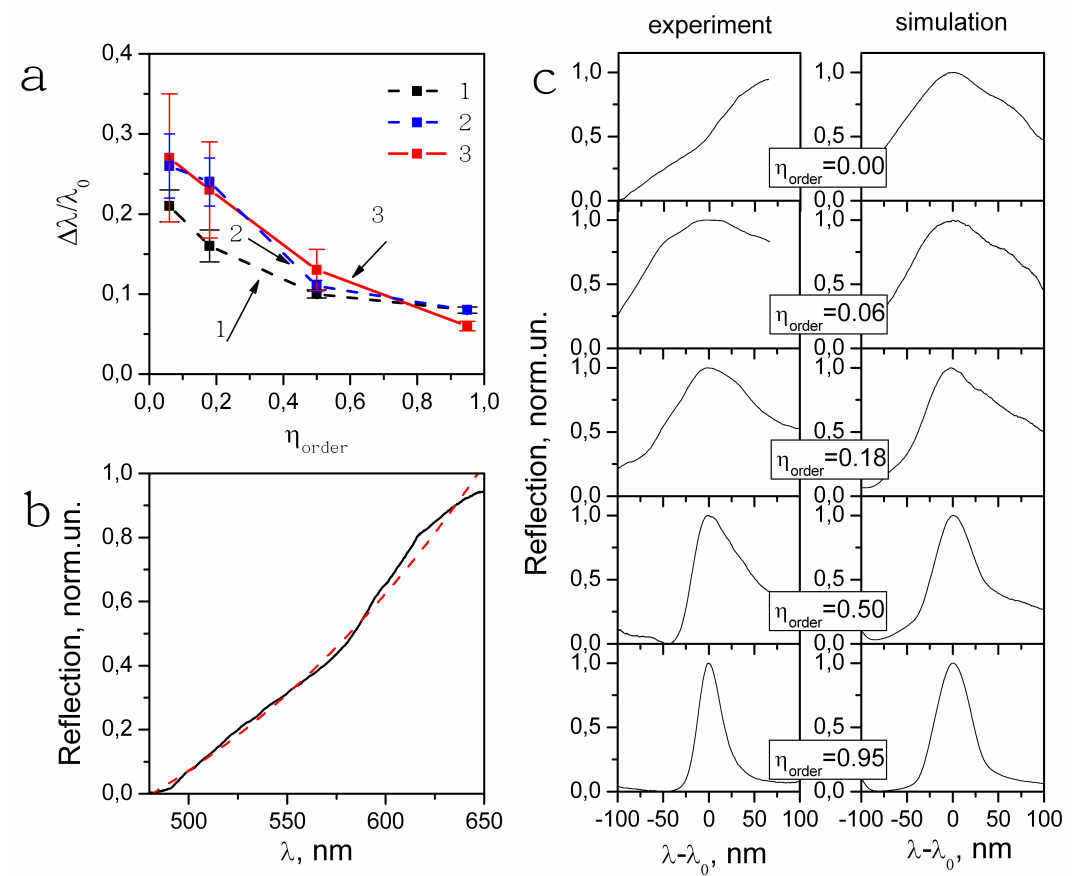

Fig. 3. (a) Dependence of relative halfwidth of the Bragg reflection band upon the $\eta_{\text {order }}$ coefficient: (1) calculations without taking the elastic multiple scattering into account, (2) calculations with taking the elastic multiple scattering into account, and (3) experiment; (b) Normal reflection spectrum for the photonic glass (solid line) and its fitting (dashed line); (c) Measured and simulated normal reflection spectra of the sample surfaces with different $\eta_{\text {order }}$ values. 
To take the scattering effects into consideration, we have measured the reflection spectrum of the photonic glass under normal incidence. Since the Bragg scattering is absent in this structure, the only effects that can be observed are elastic scattering processes. Here we assume that the absorption and inelastic scattering are eliminated in the initial samples. Fig. $3 \mathrm{~b}$ shows the reflection spectrum measured for the photonic glass and the fitted function for this spectrum. The product of this function and the simulated reflection spectra brings our model to the real conditions. Fig. $3 \mathrm{c}$ compares the simulated (including the scattering effects) reflection spectra with the experimental ones obtained for the case of normal light incidence, at appropriate $\eta_{\text {order }}$ values. The discrepancy of the experiment and the simulation at $\eta_{\text {order }}=0,00$ indicates the limit of applicability of our model, which corresponds to an extreme degree of defectiveness. Finally, curve 2 in Fig. 3a shows the dependence of $\Delta \lambda / \lambda_{0}$ on $\eta_{\text {order }}$, which probes the effect of scattering. Notice that this curve is closer to experimental one. Hence, in addition to the effects of Bragg diffraction, the effects of elastic multiple scattering can also manifest themselves in the reflection spectra.

\subsection{The influence of defects on the integral DOS spectra}

It is well known that the function of the integral DOS for the PCs has singularities at the edges of the photonic band gap, which represent a result of structural regularity. The importance of role of the DOS in the optical processes [19] makes it necessary to study the influence of structural defects on the nature of this function.

We have considered the disorder over the $d$ and $D$ values as described above in the model, resulting in the DOS plotted as a dependence on $\lambda$. Fig. 4 shows the changes in the DOS that occur with increasing $d_{\text {disamp }}$ and $D_{\text {disamp }}$. It is seen that very small deviations ( $d_{\text {disamp }}$ and $D_{\text {disamp }}=6 \mathrm{~nm}$ are not larger than $2-3 \%$ of the values $d=200 \div 220 \mathrm{~nm}$ and $D=250 \div 270 \mathrm{~nm}$ ) from the proper structural period and globule diameter leads to gradual filling of the states located in the middle of the bandgap, until the complete filling is achieved.

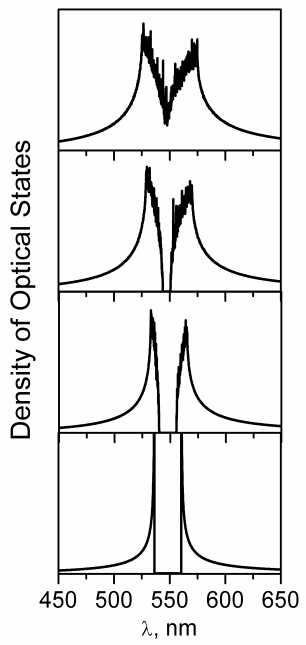

Fig. 4. Evolution of integral DOS spectrum occurring when $d_{\text {disamp }}$ and $D_{\text {disamp }}$ gradually increase (from bottom to top: $d_{\text {disamp }}$ and $D_{\text {disamp }}$ are equal to $0,2,4$ and $6 \mathrm{~nm}$ ).

Due to the microscopic defects, real SO samples always reveal deviations of the structural period (at least a few percent), whereas deviations from the normal globule diameter can be as large as at least $5 \%$. This suggests that we have studied the samples where the optical states within the photonic stop-band are completely filled. 


\section{Amplification effects in the fluorescence and Raman spectra}

Two basic mechanisms of radiation amplification can be expected in the PCs. The first is due to large-angle Bragg diffraction of short-wavelength radiation at the system of planes, with subsequent escaping of radiation along the stop-band direction [20]. The second mechanism is linked with multiple scattering of exciting photons on the structural defects of the PC, which leads to increase in the mean time of their residence in the sample volume, and consequently, their accumulation. Thus, localized modes appear on the structural defects and we have local-density enhancement of the electromagnetic field [21]. The closed paths of the source and emitted photons can arise at significant amounts of the structural defects. This can lead to the conditions under which a lasing effect appears [13].

The fluorescence spectra of the opals infiltrated with R6G have been measured in the 'reflection' geometry under absolutely identical conditions. The samples with different ordering degrees have been used. These are three samples denoted as $\mathrm{SO} 1, \mathrm{SO} 2$ and $\mathrm{SO} 3$. The concentration of R6G molecules in the infiltrated solution for all the samples has been the same $[22,23]$. The initial samples have been optically characterized before infiltration to determine the relative halfwidth $\Delta \lambda / \lambda_{0}$ of the Bragg reflection band. The relative ordering parameter $\eta_{\text {order }}$ has been determined by the $\Delta \lambda / \lambda_{0}$ value and the experimental dependence depicted in Fig. 3a.

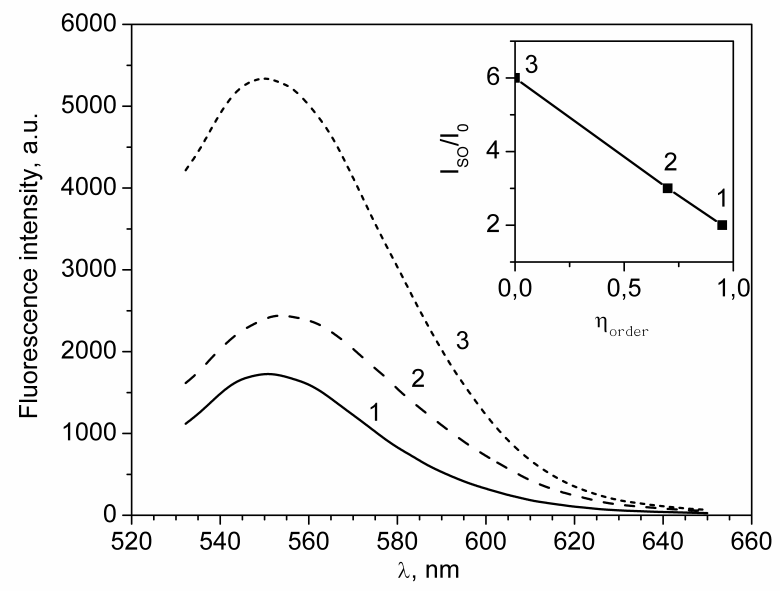

Fig. 5. Fluorescence spectra of SO samples infiltrated with R6G with different amounts of structural defects. The amount of structural defects increases with increasing label of the spectrum (1,2 and 3$)$. The insert shows dependence of relative integral intensity on the relative ordering parameter, as obtained for the corresponding samples.

The $I_{S O} / I_{0}$ value given in Fig. 5 is the ratio of the integral intensity $I_{S O}$ for R6G in the SO to the integral fluorescence intensity of R6G-ethanol solution in an optical cell. This parameter corresponds to the criterion indicating to amplification of spontaneous emission intensity in the SO. Thus, the fluorescence spectra reveal an increase in the integral luminescence intensity with increasing amount of structural defects in the opals. This increase in the intensity correlates with the increase seen for the relative ordering parameter $\eta_{\text {order }}$ and, consequently, the increase of 'slow' exciting photons with a diffusive distribution character.

We have also measured the Raman spectra for the SO filled by $\mathrm{Bi}_{12} \mathrm{SiO}_{20}$ and $\mathrm{Bi}_{12} \mathrm{SiO}_{20}$ powders . Besides of some redistribution among the Raman lines and arising of new lines (the latter can be treated as a result of crystallization in opal pores), the comparative analysis shows a 
significant (over 3 times) amplification of the integral intensity of the Raman lines (see Fig. 6). A mismatch of the Raman spectral range and the spectral position of the photonic stop-band in our samples suggest that the amplification in our case is due to the multiple scattering of excited photons.

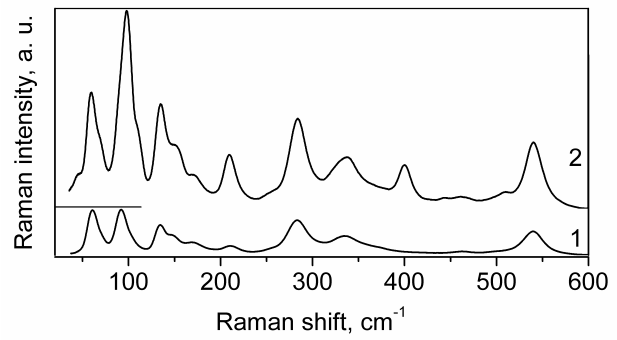

Fig. 6. Raman spectra of $\mathrm{Bi}_{12} \mathrm{SiO}_{20}$ powder (1) and opal- $\mathrm{Bi}_{12} \mathrm{SiO}_{20}$ sample (2).

\section{Conclusions}

The types of structural defects present in SO samples are classified and the relative ordering parameter of the SO structure is introduced. The reflection spectra and the DOS spectra are simulated using respectively the transfer-matrix method and the plane-wave expansion method. The relative halfwidths $\Delta \lambda / \lambda_{0}$ of the reflection band, which are dependent on the $\eta_{\text {order }}$ parameter, are compared basing on the experimental and calculated data. The best agreement between the experiment and the simulations occurs if the elastic multiple scattering effect is taken into account. It is ascertained that small (up to 3\%) deviations of the quantities $d=200 \div 220 \mathrm{~nm}$ and $D=250 \div 270 \mathrm{~nm}$ can lead to complete filling of the photon states within the photonic stopband. Amplification of the fluorescence spectra of R6G and the Raman spectra of $\mathrm{Bi}_{12} \mathrm{SiO}_{20}$ incorporated in the SO templates is observed.

\section{Acknowledgments}

This work was supported by the Ukrainian-Russian project 27-02-14 RFFD "Capture of electromagnetic emission in resonance structures and in resonance photonic crystals with luminescent centres".

\section{References}

1. Sinitskii A, Knot'ko A and Tretyakov Yu, 2004. Silica photonic crystals: synthesis and optical properties. Sol. State Ion. 172: 477-479.

2. Vlasov Y, Bo X, Sturm J and Norris D, 2001. On-chip natural assembly of silicon photonic bandgap crystals. Nature. 414: 289-293.

3. García-Santamaría F and Braun P, 2007. Are artificial opals non-close-packed fcc structures? App. Phys. Lett. 90: 241905-1-241905-3.

4. Joannopoulos J, Villeneuve P and Fan S, 1997. Photonic crystals: putting a new twist on light. Nature. 386: 143-149.

5. Sinitskii A, Abramova V, Laptinskaya $\mathrm{T}$ and Tretyakov $\mathrm{Yu}, 2007$. Domain mapping of inverse photonic crystals by laser diffraction. Phys. Lett. A. 366: 516-522.

6. Baryshev A, Kaplyanskii A, Kosobukin V, Limonov M, Samusev K and Usvyat D, 2003. Bragg diffraction of light in synthetic opals. Phys. Sol. State. 45: 459-471.

7. Kaliteevskii M, Nikolayev V and Abram R, 2005. Eigenstate statistics and optical properties of one-dimensional disordered photonic crystals. Phys. Sol. State. 47: 1948-1957.

8. Zheltikov A, Magnitskii S and Tarasishin A, 1999. Localization and channeling of light in 
defect modes of two-dimensional photonic crystals. JETP Lett. 70: 323-328.

9. Lončar M, Nedeljković D, Pearsall T, Vučković J, Scherer A, Kuchinsky S and Allan D, 2002. Experimental and theoretical confirmation of Bloch-mode light propagation in planar photonic crystal waveguides. Appl. Phys. Lett. 80: 1689-1691.

10. Inoue $\mathrm{K}$ and Ohtaka $\mathrm{K}$. Photonic crystals: physics, fabrication and application. Berlin: Springer (2004).

11. John S, 1987. Strong localization of photons in certain disordered dielectric superlattices. Phys. Rev. Lett. 58: 2486-2489.

12. John S, 1991. Localization of light. Phys. Today. 44: 32-40.

13. Gottardo S, Sapienza R, Garcia P, Blanco A, Wiersma D and López C, 2008. Resonancedriven random lasing. Nature Photon. 2: 429-432.

14. Stöber W, Fink A and Bohn E, 1968. Controlled growth of monodisperse silica spheres in micron size range. J. Coll. Inter. Sci. 26: 62-68.

15. Born M and Wolf E. Principles of optics. Oxford: Pergamon Press (1970).

16. Yariv A and Yeh P. Optical waves in crystals. New York: Wiley (1984).

17. Sakoda K Optical properties of photonic crystals, $2^{\text {nd }}$ ed. Heidelberg: Springer (2005).

18. Joannopoulos J, Johnson S, Winn J and Meade R. Photonic crystals: molding the flow of light. Princeton and Oxford: Princeton University Press (2008).

19. Gaponenko S. Introduction to nanophotonics. Cambridge: Cambridge University Press (2010).

20. Bechger L, Lodahl P and Vos W, 2005. Directional fluorescence spectra of laser dye in opal and inverse opal photonic crystals. J. Phys. Chem. B. 109: 9980-9988.

21. Spitsyn A and Glinskii G, 2008. Computation of localized modes in a defect-containing photonic crystal by the method of periodic continuation of solution. Techn. Phys. 53: 602608.

22. Moiseyenko V, Dergachov M, Shvachich V and Yevchik A, 2009. The possibility for surfaceenhanced Raman scattering and spontaneous parametric down-conversion by globular photonic crystals infiltrated with dielectrics. Ukr. J. Phys. Opt. 10: 201-205.

23. Moiseyenko V and Dergachov M. Quantum optics phenomena in synthetic opal photonic crystals. Quantum optics and laser experiments. Rijeka: InTech (2012).

Yevchik A., Moiseyenko V. and Dergachov M. 2015. The influence of structural defects on the optical properties of synthetic opals. Ukr.J.Phys.Opt. 16: $24-31$.

Анотація. Досліджено вплив дефектів структури опалів на спектри брегівського відбивання, флюоресценції та комбінаційного розсіяння світла. Спираючись на структуру реальних опалів, запропоновано відносний параметр упорядкованості як критерій якості зразків. Розроблено одновимірну модель періодичної шаруватої структури, що включає типові для синтетичних опалів дефекти. На підставі порівняння експериментальних $i$ розрахованих спектрів брегівського відбивання показано, що модель може стати більи наближеною до реальних умов, якщо врахувати інтерференцію світла в об'ємі опала в умовах багатократного пружного розсіювання. Для вивчення впливу дефектів структури на спонтанне випромінювання, а також на процеси непружного розсіювання світла виміряно спектри флуоресиениіï барвника R6G і спектри комбінаційного розсіювання світла активного діелектрика $\mathrm{Bi}_{12} \mathrm{SiO}_{20}$ у порах синтетичного опалу. Виявлено ефекти підсилення інтенсивності смуг у спектрах флюоресценщії R6G і спектрах комбінаційного розсіювання світла $\mathrm{Bi}_{12} \mathrm{SiO}_{20}$, якщо порівнювати зі спектрами цих речовин у вільному стані. 